\title{
On long-step surrogate projection methods for solving convex feasibility problems
}

\author{
Krzysztof C. Kiwiel, Bożena Lopuch \\ Systems Research Institute \\ Newelska 6, 01-447 Warsaw, Poland. \\ e-mail: kiwiel@ibspan.waw.pl and lopuch@ibspan.waw.pl
}

\begin{abstract}
We present methods for finding common points of finitely many closed convex sets in a Hilbert space. Every iteration approximates each set by a halfspace given by an approximate projection of the current iterate. The next iterate is found by projecting the current one on a surrogate halfspace formed by taking a convex combination of the halfspace inequalities. The resulting methods are block-iterative and hence lend themselves to parallel implementation. We extend to accelerated methods some recent results of Bauschke and Borwein on convergence of projection methods.
\end{abstract}

\section{Keywords}

Successive projections, relaxation methods, convex feasibility problems, surrogate inequalities.

\section{INTRODUCTION}

Let $\left\{C_{i}\right\}_{i=1}^{N}$ be a collection of closed convex subsets of a real Hilbert space $\mathcal{H}$ with inner product $\langle\cdot, \cdot\rangle$ and norm $\|\cdot\|$. Suppose $\cap_{i=1}^{N} C_{i} \neq \emptyset$. Consider the convex feasibility problem (CFP)

find any point $x$ in $C:=\cap_{i=1}^{N} C_{i}$.

Kiwiel (1995a) has recently studied long-step surrogate projection methods for CFP's in finite-dimensional spaces, motivated mainly by their possible applications in methods for convex nondifferentiable optimization (Kiwiel, 1995b). We now give convergence and rate of convergence results in the infinite-dimensional setting. Such results indicate that our methods should work well even when the dimension of the space becomes very large. Our results extend to long-step methods some results of Bauschke and Borwein (1995) concerning short-step methods, which in turn generalize those in (Aharoni and Censor,

Research supported by the Polish State Committee for Scientific Research under Grants 8S50502206 and $8 \mathrm{~T} 11 \mathrm{~A} 00409$. 
1989; Flåm and Zowe, 1990); see (Bauschke and Borwein, 1995) for more references and an extensive list of applications of CFP's.

A general surrogate projection method is described in $\S 2$. In $\S \S 3-4$ we give a sample of results on convergence and rate of convergence that supplement those in Kiwiel (1995a). For brevity, we omit the proofs given in (Kiwiel and Lopuch, 1994).

A set of the form $H=\{x:\langle a, x\rangle \leq b\}$, where $a \in \mathcal{H}$ and $b \in \mathbb{R}$, is called a halfspace, including $H=\mathcal{H}$ or $\emptyset$. Given a closed convex set $D, P_{D} x=\arg \min _{y \in D}\|x-y\|$ is the projector on $D$ and $d_{D}=\inf _{y \in D}\|\cdot-y\|$ is the distance function of $D$. int $C$ denotes the interior of $C$. We let $\{1: N\}=\{1,2, \ldots, N\}$ and $\mathbb{R}_{+}^{N}=\left\{\lambda \in \mathbb{R}^{N}: \lambda \geq 0\right\}$. We need

Lemma 1 If $D$ is a closed convex set and $\alpha \geq 0$ then $R_{D, \alpha}=(1-\alpha) I+\alpha P_{D}$ satisfies $\left\|c-R_{D, \alpha} x\right\|^{2} \leq\|c-x\|^{2}-\alpha(2-\alpha)\left\|x-P_{D} x\right\|^{2} \forall c \in D, x \in \mathcal{H}$.

\section{A GENERAL ALGORITHM AND ITS BASIC PROPERTIES}

We first state a general algorithm for (1).

\section{Algorithm 2}

Step 0 (Initiation). Select an initial $x^{0} \in \mathcal{H}$ and a weight threshold $\lambda_{\min } \in\left(0, \frac{1}{N}\right]$. Set $n=0$.

Step 1 (Working set selection). Choose a nonempty set $\hat{I}^{(n)} \subset\{1: N\}$, so that for each $i \in\{1: N\}, i \in \hat{I}^{(n)}$ for infinitely many $n$.

Step 2 (Halfspace selection). For each $i \in \hat{I}^{(n)}$, choose a projection operator $P_{i}^{(n)}: \mathcal{H} \rightarrow \mathcal{H}$ with $P_{i}^{(n)} \mathcal{H} \supset C_{i}$, let $x^{i n}=P_{i}^{(n)} x^{n}$ and $H_{i}^{n}=\left\{x:\left\langle a^{i n}, x\right\rangle \leq b_{i n}\right\}$ with

$\left(a^{i n}, b_{i n}\right)=\left(x^{n}-x^{i n},\left\langle x^{n}-x^{i n}, x^{i n}\right\rangle\right), \quad i \in \hat{I}^{(n)}$.

Step 3 (Surrogate construction). Find a weight vector $\lambda^{n} \in \mathbb{R}_{+}^{N}, \lambda_{i}^{n}=0, i \notin \hat{I}^{(n)}$, $\sum_{i=1}^{N} \lambda_{i}^{n}=1$, for the surrogate inequality $\left\langle\hat{a}^{n}, x\right\rangle \leq \hat{b}_{n}$ with

$\left(\hat{a}^{n}, \hat{b}_{n}\right)=\sum_{i \in \hat{I}^{(n)}} \lambda_{i}^{n}\left(a^{i n}, b_{i n}\right)=\sum_{i \in \hat{I}^{(n)}} \lambda_{i}^{n}\left(x^{n}-x^{i n},\left\langle x^{n}-x^{i n}, x^{i n}\right\rangle\right)$

such that the surrogate halfspace $\hat{H}^{n}=\left\{x:\left\langle\hat{a}^{n}, x\right\rangle \leq \hat{b}_{n}\right\}$ satisfies

$d_{\hat{H}^{n}}\left(x^{n}\right) \geq \lambda_{\min } \max _{i \in \hat{I}^{(n)}} d_{H_{i}^{n}}\left(x^{n}\right)$.

Step 4 (Relaxation). Select a relaxation parameter $\alpha^{(n)} \in(0,2]$ and set (cf. Lem. 1)

$x^{n+1}=R_{\hat{H}^{n}, \alpha^{(n)}} x^{n}=x^{n}+\alpha^{(n)}\left(P_{\hat{H}^{n}} x^{n}-x^{n}\right)$,

$\sigma_{n}=\alpha^{(n)}\left(2-\alpha^{(n)}\right) d_{\hat{H}^{n}}^{2}\left(x^{n}\right)$.

Step 5. Increase $n$ by 1 and go to Step 1 . 
At Step 2, by Lem. 1, $C_{i} \subset P_{i}^{(n)} \mathcal{H} \subset H_{i}^{n}, \forall i \in \hat{I}^{(n)}$. Let $I^{(n)}=\left\{i: \lambda_{i}^{n}>0\right\}$. By (2)-(3), $\left\langle a^{i n}, x^{n}\right\rangle-b_{i n}=\left\|a^{i n}\right\|^{2}=\left\|x^{n}-x^{i n}\right\|^{2} \quad \forall i \in \hat{I}^{(n)}$,

$\left\langle\hat{a}^{n}, x^{n}\right\rangle-\hat{b}_{n}=\sum_{i \in I^{(n)}} \lambda_{i}^{n}\left\|x^{n}-x^{i n}\right\|^{2} \quad$ and $\quad\left\|\hat{a}^{n}\right\|=\left\|\sum_{i \in I^{(n)}} \lambda_{i}^{n}\left(x^{n}-x^{i n}\right)\right\|$,

so $x^{i n}=P_{H_{i}^{n}}\left(x^{n}\right), d_{H_{i}^{n}}\left(x^{n}\right)=\left\|x^{n}-x^{i n}\right\|=\left\|a^{i n}\right\|, i \in \hat{I}^{(n)}$. At Step 3, (4) holds if $\lambda_{i}^{n} \geq \lambda_{\min }$ for some $\hat{\imath} \in \operatorname{Arg} \max _{i \in \hat{I}(n)}\left\|x^{n}-x^{i n}\right\|$, since

$d_{\hat{H}^{n}}\left(x^{n}\right)=\frac{\left\langle\hat{a}^{n}, x^{n}\right\rangle-\hat{b}_{n}}{\left\|\hat{a}^{n}\right\|} \geq \lambda_{\min } \frac{\max _{i \in \hat{I}^{(n)}}\left(\left\langle a^{i n}, x^{n}\right\rangle-b_{i n}\right)}{\max _{i \in \hat{I}^{(n)}}\left\|a^{i n}\right\|}=\lambda_{\min } \max _{i \in \hat{I}^{n}} d_{H_{i}^{n}}\left(x^{n}\right)$

from $\left\langle\hat{a}^{n}, x^{n}\right\rangle-\hat{b}_{n} \geq \lambda_{\min }\left\|a^{i n}\right\|^{2}$ and $\left\|\hat{a}^{n}\right\| \leq \sum_{i} \lambda_{i}^{n} \max _{i}\left\|a^{i n}\right\|$ by the convexity of $\|\cdot\|$. Since $\cap_{i \in I^{(n)}} C_{i} \subset \cap_{i \in I^{(n)}} H_{i}^{n} \subset \hat{H}^{n}$ from $\lambda^{n} \geq 0$, by Lem. 1 and (6),

$\left\|c-x^{n+1}\right\|^{2} \leq\left\|c-x^{n}\right\|^{2}-\alpha^{(n)}\left(2-\alpha^{(n)}\right) d_{\hat{H}^{n}}^{2}\left(x^{n}\right)=\left\|c-x^{n}\right\|^{2}-\sigma_{n} \quad \forall c \in \cap_{i \in I^{(n)}} C_{i}$.

Thus progress towards the solution is measured by $d_{\hat{H}^{n}}\left(x^{n}\right)$, and one is interested in deep cuts that have $d_{\hat{H}^{n}}\left(x^{n}\right)$ as large as possible (Kiwiel, 1995a).

Remark 3 Other ways of finding cuts are given in (Kiwiel, 1995a). First, the weights $\lambda_{i}^{n}=\left\|a^{i n}\right\| \gamma / \sum_{j}\left\|a^{j n}\right\|^{\gamma}, i \in \hat{I}^{(n)}$, are admissible if $\gamma \geq 0$, since $\max _{i \in I_{\max }^{(n)}} \lambda_{i}^{n} \geq 1 /\left|\hat{I}^{(n)}\right|$; e.g., $\gamma=0$ gives $\lambda_{i}^{n}=1 /\left|I_{+}^{(n)}\right|, i \in I_{+}^{(n)}:=\left\{i: a^{i n} \neq 0\right\}$. Second, the deepest surrogate cut that maximizes $d_{\hat{H}^{n}}\left(x^{n}\right)$ is obtained for weights that solve (cf. (7)-(8))

$\max \left\{\frac{\sum_{i \in \hat{I}^{(n)}} \lambda_{i}\left\|a^{i n}\right\|^{2}}{\left\|\sum_{i \in \hat{I}^{(n)}} \lambda_{i} a^{i n}\right\|}: \lambda_{i} \geq 0, i \in \hat{I}^{(n)}, \sum_{i \in \hat{I}^{(n)}} \lambda_{i}=1\right\}$,

in which case $P_{\hat{H}^{n}} x^{n}=P_{\cap_{i \in \hat{I}(n)} H_{i}^{n}} x^{n}$. Approximate solutions to (10) (that satisfy (4) with $\lambda_{\min }=1$ ) are discussed in (Kiwiel, 1995a, $§ 8$ ).

Remark 4 To compare Algorithm 2 with that of Bauschke and Borwein (1995), let

$\hat{c}_{n}=\left(\left\langle\hat{a}^{n}, x^{n}\right\rangle-\hat{b}_{n}\right) /\left\|\hat{a}^{n}\right\|^{2}$.

Then

$$
\begin{aligned}
& P_{\hat{H}^{n}}\left(x^{n}\right)-x^{n}=-\hat{c}_{n} \hat{a}^{n}=\frac{\sum_{i \in I^{(n)}} \lambda_{i}^{n}\left\|x^{n}-x^{i n}\right\|^{2}}{\left\|x^{n}-\sum_{i \in I^{(n)}} \lambda_{i}^{n} x^{i n}\right\|^{2}}\left(\sum_{i \in I^{(n)}} \lambda_{i}^{n} x^{i n}-x^{n}\right), \\
& d_{\hat{H}^{n}}\left(x^{n}\right)=\frac{\left\langle\hat{a}^{n}, x^{n}\right\rangle-\hat{b}_{n}}{\left\|\hat{a}^{n}\right\|}=\frac{\sum_{i \in I^{(n)}} \lambda_{i}^{n}\left\|x^{n}-x^{i n}\right\|^{2}}{\left\|\sum_{i \in I^{(n)}} \lambda_{i}^{n}\left(x^{n}-x^{i n}\right)\right\|}
\end{aligned}
$$


and $x^{n+1}=x^{n}-\alpha^{(n)} \hat{c}_{n} \hat{a}^{n}$ if $\hat{a}^{n} \neq 0$ (cf. (3), (5), (8)). The method of Bauschke and Borwein (1995) would produce

$\tilde{x}^{n+1}=\sum_{i \in I^{n}} \lambda_{i}^{n} R_{H_{i}^{n}, \alpha^{(n)}}\left(x^{n}\right)=x^{n}+\alpha^{(n)}\left(\sum_{i \in I^{n}} \lambda_{i}^{n} x^{i n}-x^{n}\right)$

instead of $x^{n+1}$. To see why this method works, apply Lem. 1 to obtain

$\left\|c-R_{H_{i}^{n}, \alpha^{(n)}}\left(x^{n}\right)\right\|^{2} \leq\left\|c-x^{n}\right\|^{2}-\alpha^{(n)}\left(2-\alpha^{(n)}\right)\left\|x^{n}-x^{i n}\right\|^{2} \quad \forall c \in H_{i}^{n} \supset C_{i} ;$

multiply this by $\lambda_{i}^{n}$, sum over $i$ and use $\sum_{i} \lambda_{i}^{n}=1$ and the convexity of $\|\cdot\|^{2}$ to get

$\left\|c-\tilde{x}^{n+1}\right\|^{2} \leq\left\|c-x^{k}\right\|^{2}-\alpha^{(n)}\left(2-\alpha^{(n)}\right) \sum_{i \in I^{(n)}} \lambda_{i}^{n}\left\|x^{n}-x^{i n}\right\|^{2} \quad \forall c \in \bigcap_{i \in I^{(n)}} C_{i}$.

Thus to compare the methods (5) and (14) via their Fejér estimates (9) and (15), we may use the ratio of $\sigma_{n}$ (given by (6) and (13)) and (cf. (8))

$\tilde{\sigma}_{n}=\alpha^{(n)}\left(2-\alpha^{(n)}\right) \sum_{i \in I^{(n)}} \lambda_{i}^{n}\left\|x^{n}-x^{i n}\right\|^{2}=\alpha^{(n)}\left(2-\alpha^{(n)}\right)\left(\left\langle\hat{a}^{n}, x^{n}\right\rangle-\hat{b}_{n}\right)$.

Lemma 5 Let $\tilde{x}^{n+1}=x^{n}-\alpha^{(n)} \hat{a}^{n}$ denote the point produced by the iteration (14) (cf. (3)), and let $x^{n+1}$ be given by (5). Then:

(i) $x^{n} \in \hat{H}^{n} \Longleftrightarrow\left\langle\hat{a}^{n}, x^{n}\right\rangle=\hat{b}_{n} \Longleftrightarrow \sum_{i \in I^{(n)}} \lambda_{i}^{n}\left\|x^{n}-x^{i n}\right\|^{2}=0 \Longleftrightarrow x^{n} \in H_{i}^{n}$ $\forall i \in I^{(n)} \Longleftrightarrow \tilde{\sigma}_{n}=0 \Longleftrightarrow \sigma_{n}=0$. Moreover, each of these conditions implies $\hat{a}^{n}=0$ and $x^{n+1}=\tilde{x}^{n+1}=x^{n}$.

(ii) If $\cap_{i \in I^{(n)}} H_{i}^{n} \neq \emptyset$ then $\hat{a}^{n}=0 \Longleftrightarrow \hat{H}^{n}=\mathcal{H} \Longleftrightarrow$ all the conditions of (i) hold.

(iii) If $\hat{a}^{n} \neq 0$ then $x^{n+1}=x^{n}-\alpha^{(n)}\left(\sigma_{n} / \tilde{\sigma}_{n}\right) \hat{a}^{n}$, where

$$
\begin{aligned}
& \sigma_{n} / \tilde{\sigma}_{n}=\hat{c}_{n}=\frac{\left\langle\hat{a}^{n}, x^{n}\right\rangle-\hat{b}_{n}}{\left\|\hat{a}^{n}\right\|^{2}}=\frac{\sum_{i \in I^{(n)}} \lambda_{i}^{n}\left\|x^{n}-x^{i n}\right\|^{2}}{\left\|\sum_{i \in I^{(n)}} \lambda_{i}^{n}\left(x^{n}-x^{i n}\right)\right\|^{2}} \geq 1, \\
& \text { and } \sigma_{n}=\tilde{\sigma}_{n} \Longleftrightarrow x^{i n}=x^{j n} \forall i, j \in I^{(n)}
\end{aligned}
$$

(iv) If $\alpha^{(n)}=1$ then $x^{n+1} \in \hat{H}^{n}$, whereas $\tilde{x}^{n+1} \in \hat{H}^{n} \Longleftrightarrow \tilde{\sigma}_{n}=\sigma_{n}$.

We note that both the long-step method (5) and the short-step method (14) use the same direction negative to the normal of the surrogate inequality. Lemma 5 says that the long-step method can take a much longer step in this direction, thus providing a much larger guaranteed progress when $\sigma_{n} \gg \tilde{\sigma}_{n}$ in (9) and (15) (i.e., some tangent cone to $\cap_{i \in J^{k}} H_{i}^{k}$ is 'flat').

\section{BASIC CONVERGENCE RESULTS}

Let $\underline{\alpha}^{(\infty)}=\underline{\lim }_{n} \alpha^{(n)}$ and $\bar{\alpha}^{(\infty)}=\varlimsup_{n} \alpha^{(n)}$.

Lemma 6 (i) $\left\|c-x^{n}\right\|^{2}-\left\|c-x^{n+1}\right\|^{2} \geq \alpha^{(n)}\left(2-\alpha^{(n)}\right) d_{\hat{H}^{n}}^{2}\left(x^{n}\right)$ if $c \in \cap_{i \in I^{(n)}} C_{i}$.

(ii) $\left\|c-x^{n}\right\|^{2}-\left\|c-x^{m}\right\|^{2} \geq \sum_{l=n}^{m-1} \alpha^{(l)}\left(2-\alpha^{(l)}\right) d_{\hat{H}^{l}}^{2}\left(x^{l}\right)$ if $c \in \cap_{l=n}^{m-1} \cap_{i \in I^{(l)}} C_{i}, m \geq n \geq 0$.

(iii) $\left\{x^{n}\right\}$ is Fejér monotone w.r.t. $C$ (hence bounded) and $\sum_{l=0}^{\infty} \alpha^{(l)}\left(2-\alpha^{(l)}\right) d_{\hat{\boldsymbol{H}}^{l}}^{2}\left(\bar{x}^{l}\right)<\infty$. 
Corollary 7 (i) If int $C \neq \emptyset$ then $\left\{x^{n}\right\}$ converges in norm to some $x \in \mathcal{H}$. (ii) If $\varliminf_{n} d_{C}\left(x^{n}\right) \rightarrow 0$, then $\left\{x^{n}\right\}$ converges in norm to some $x \in C$.

Corollary 8 If int $C \neq \emptyset$ then $\sum_{n}\left\|x^{n+1}-x^{n}\right\|<\infty$ and $\sum_{n} \alpha^{(n)} d_{\hat{H}^{n}}\left(x^{n}\right)<\infty$.

Corollary 9 If $\bar{\alpha}^{(\infty)}<2$ then the algorithm is regular, i.e., $\left\|x^{n+1}-x^{n}\right\| \rightarrow 0$.

Further assumptions are necessary to ensure that the algorithm converges.

Definition 10 We say the algorithm is focusing if for each index $i$ and subsequence $\left\{x^{n_{k}}\right\}$, the conditions $x^{n_{k}}-x, d_{H_{i}^{n_{k}}}\left(x^{n_{k}}\right) \rightarrow 0$ (i.e., $x^{n_{k}}-P_{i}^{\left(n_{k}\right)} x^{n_{k}} \rightarrow 0$ ) and $i \in I^{\left(n_{k}\right)} \forall k$ imply $x \in C_{i}$, where $\rightarrow$ and $\rightarrow$ stand for norm and weak convergence respectively.

Remark 11 The above definition of Bauschke and Borwein (1995) is equivalent to the concept of cut map introduced by Kiwiel (1995a) when $\operatorname{dim} \mathcal{H}<\infty$.

Theorem 12 Suppose the algorithm is focusing. If $0<\underline{\alpha}^{(\infty)}, \bar{\alpha}^{(\infty)}<2$ then $\left\{x^{n}\right\}$ either converges in norm to some point in $C$ or has no norm cluster points at all.

Remark 13 If there exists $\epsilon>0$ s.t. $\left\{\alpha^{(n)}\right\} \subset[\epsilon, 2-\epsilon]$ then $0<\underline{\alpha}^{(\infty)}, \bar{\alpha}^{(\infty)}<2$.

For motivation, we quote the following result of Bauschke and Borwein (1995).

Proposition 14 Suppose for every index $i,\left\{P_{i}^{(n)}\right\}$ converges actively pointwise to $P_{C_{i}}$, i.e., $\lim _{n: i \in I^{(n)}} P_{i}^{(n)} x=P_{C_{i}} x$ for every $x \in \mathcal{H}$. Then the algorithm is focusing.

Definition 15 If there is a positive integer $p$ s.t. $i \in \hat{I}^{(n)} \cup \hat{I}^{(n+1)} \cup \cdots \hat{I}^{(n+p-1)}$ for every index $i$ and all $n$, then we speak of an intermittent or $p$-intermittent algorithm.

Theorem 16 (i) Suppose the algorithm is focusing and intermittent, and $0<\underline{\alpha}^{(\infty)}$ and $\bar{\alpha}^{(\infty)}<2$. Then $\left\{x^{n}\right\}$ is regular and converges weakly to some point in $C$.

(ii) Suppose the algorithm is focusing, p-intermittent, regular (cf. Cor. 9) and $\hat{\nu}_{n}=$ $\min \left\{\alpha^{(l)}\left(2-\alpha^{(l)}\right): n p \leq l<(n+1) p\right\} \forall n \geq 0$. If $\sum_{n} \hat{\nu}_{n}=\infty$ then $\left\{x^{n}\right\}$ has a unique weak cluster point in $C$. In particular, this happens whenever $0<\underline{\alpha}^{(\infty)}, \bar{\alpha}^{(\infty)}<2$.

(iii) Suppose the algorithm is focusing and $x^{n} \rightarrow x$. If $\sum_{n: i \in \hat{I}^{(n)}} \alpha^{(n)}\left(2-\alpha^{(n)}\right)=\infty$ for some index $i$, then $x \in C_{i}$. Consequently, $x \in C$ if $\sum_{n: i \in \hat{I}^{(n)}} \alpha^{(n)}\left(2-\alpha^{(n)}\right)=\infty$ for every index $i$.

Corollary 17 Suppose the algorithm is focusing, $\sum_{n: i \in \hat{I}^{(n)}} \alpha^{(n)}\left(2-\alpha^{(n)}\right)=\infty$ for every index $i$ and $\operatorname{int} C \neq \emptyset$. Then $\left\{x^{n}\right\}$ converges in norm to some point in $C$.

Remark 18 If $0<\underline{\alpha}^{(\infty)}, \bar{\alpha}^{(\infty)}<2$ then $\sum_{n: i \in \hat{I}^{(n)}} \alpha^{(n)}\left(2-\alpha^{(n)}\right)=\infty$ for all $i$.

Theorem 19 Suppose the algorithm is focusing.

(i) If $\sum_{n: \tilde{I}^{(n)}=\{1: N\}} \alpha^{(n)}\left(2-\alpha^{(n)}\right)=\infty$ then $\left\{x^{n}\right\}$ converges weakly to some point in $C$. 
(ii) If there exist $\epsilon>0$ and a subsequence $\left\{x^{n_{k}}\right\}$ s.t. $\epsilon \leq \alpha^{\left(n_{k}\right)} \leq 2-\epsilon$ and $\hat{I}^{\left(n_{k}\right)}=\{1: N\}$ for all $k$ then $x^{n} \rightarrow x$ for some $x \in C$.

Definition 20 We say the algorithm is linearly focusing if there is $\beta>0$ s.t. $\beta d_{C_{i}}\left(x^{n}\right) \leq$ $d_{H_{i}^{n}}\left(x^{n}\right)$ for all large $n$ and every $i \in \hat{I}^{(n)}$; it is strongly focusing if for every index $i$ and every subsequence $\left\{x^{n_{k}}\right\}$, the conditions $x^{n_{k}} \rightarrow x, d_{H_{i}^{n}}\left(x^{n_{k}}\right) \rightarrow 0$ and $i \in \hat{I}^{\left(n_{k}\right)}$ imply $d_{C_{i}}\left(x^{n_{k}}\right) \rightarrow 0$. Thus (cf. Def. 10): linearly focusing $\Rightarrow$ strongly focusing $\Rightarrow$ focusing.

Corollary 21 Suppose the algorithm is linearly focusing, $0<\underline{\alpha}^{(\infty)}, \bar{\alpha}^{(\infty)}<2$ and either $\mathcal{H}$ is finite-dimensional or int $C \neq \emptyset$. Then $\left\{x^{n}\right\}$ converges in norm to some point in $C$.

Theorem 22 Suppose the algorithm is focusing and int $C \neq \emptyset$, so that $\left\{x^{n}\right\}$ converges to some $x$. If $\sum_{n: i \in \hat{I}^{(n)}} \alpha^{(n)}=\infty$ for some index $i$, then $x \in C_{i}$. Consequently, $x \in C$ if $\sum_{n: i \in \hat{I}^{(n)}} \alpha^{(n)}=\infty$ for every index $i$.

Definition 23 We say the algorithm considers remotest sets if $\hat{I}^{(n)}$ contains some active remotest index $i_{n} \in \hat{I}_{\text {rem }}^{(n)}:=\left\{i: d_{C_{i}}\left(x^{n}\right)=\max _{j=1}^{N} d_{C_{j}}\left(x^{n}\right)\right\}$, for all $n$.

Theorem 24 Suppose the algorithm is strongly focusing and considers remotest sets.

(i) If $\sum_{n} \alpha^{(n)}\left(2-\alpha^{(n)}\right)=\infty$ then $\left\{x^{n}\right\}$ converges weakly to some point in $C$.

(ii) If $0<\underline{\alpha}^{(\infty)}, \bar{\alpha}^{(\infty)}<2$ then $x^{n}-x$ for some $x \in C$ and $\max _{j=1}^{N} d_{C_{j}}\left(x^{n}\right) \rightarrow 0$.

\section{NORM CONVERGENCE AND BOUNDED REGULARITY}

Guaranteeing norm convergence requires further assumptions.

Definition 25 The $N$-tuple $\left(C_{1}, \ldots, C_{N}\right)$ is regular if $\forall \epsilon>0 \exists \delta>0 \forall x \in\{x \in \mathcal{H}$ : $\left.\max _{j=1}^{N} d_{C_{j}}(x) \leq \delta\right\}: d_{C}(x) \leq \epsilon$. It is boundedly regular if this holds only on bounded sets, i.e., $\forall$ bounded $S \subset \mathcal{H} \forall \epsilon>0 \exists \delta>0 \forall x \in\left\{x \in S: \max _{j=1}^{N} d_{C_{j}}(x) \leq \delta\right\}: d_{C}(x) \leq \epsilon$.

Theorem 26 Suppose the algorithm is strongly focusing, p-intermittent, regular (cf. Cor. 9) and $\hat{\nu}_{n}=\min \left\{\alpha^{(l)}\left(2-\alpha^{(l)}\right): n p \leq l<(n+1) p\right\} \forall n \geq 0$. If $\sum_{n_{n}} \hat{\nu}_{n}=\infty$ and $\left(C_{1}, \ldots, C_{N}\right)$ is boundedly regular then $\left\{x^{n}\right\}$ converges in norm to some point in $C$. In particular, this happens whenever $0<\underline{\alpha}^{(\infty)}, \bar{\alpha}^{(\infty)}<2$.

Theorem 27 Suppose the algorithm is strongly focusing and considers remotest sets, and $\left(C_{1}, \ldots, C_{N}\right)$ is boundedly regular. If $\sum_{n} \alpha^{(n)}\left(2-\alpha^{(n)}\right)=\infty$ then $\left\{x^{n}\right\}$ converges in norm to some point in $C$. In particular, this happens whenever $0<\underline{\alpha}^{(\infty)}, \bar{\alpha}^{(\infty)}<2$.

As in (Bauschke and Borwein, 1995), guaranteeing linear convergence requires linear regularity.

Definition 28 The $N$-tuple $\left(C_{1}, \ldots, C_{N}\right)$ is called linearly regular if $\exists \kappa>0 \forall x \in \mathcal{H}$ : $d_{C}(x) \leq \kappa \max _{j=1}^{N} d_{C_{j}}(x)$. It is called boundedly linearly regular if $\forall$ bounded $S \subset \mathcal{H}$ $\exists \kappa_{S}>0 \forall x \in S: d_{C}(x) \leq \kappa_{S} \max _{j=1}^{N} d_{C_{j}}(x)$. 
We say $\left\{x^{n}\right\}$ converges linearly with rate $\beta \in[0,1)$ if there are $x \in \mathcal{H}$ and $\alpha \geq 0$ s.t. $\left\|x^{n}-x\right\| \leq \alpha \beta^{n}$ for all $n$.

Theorem 29 Suppose the algorithm is linearly focusing and p-intermittent, $\left\{\alpha^{(n)}\right\} \subset$ $[\epsilon, 2-\epsilon]$ for some $\epsilon>0$ and $\left(C_{1}, \ldots, C_{N}\right)$ is boundedly linearly regular. Then $\left\{x^{n}\right\}$ converges linearly to some point in $C$; the rate of convergence is independent of the starting point whenever $\left(C_{1}, \ldots, C_{N}\right)$ is linearly regular.

Theorem 30 Suppose the algorithm is linearly focusing, considers remotest sets, $0<$ $\underline{\alpha}^{(\infty)}, \bar{\alpha}^{(\infty)}<2$ and $\left(C_{1}, \ldots, C_{N}\right)$ is boundedly linearly regular. Then $\left\{x^{n}\right\}$ converges linearly to some point in $C$; the rate of convergence is independent of the starting point whenever $\left(C_{1}, \ldots, C_{N}\right)$ is linearly regular.

Theorem 31 Suppose the algorithm is strongly focusing and $\left(C_{1}, \ldots, C_{N}\right)$ is boundedly regular. If $\sum_{n: \hat{I}^{(n)}=\{1: N\}} \alpha^{(n)}\left(2-\alpha^{(n)}\right)=\infty$ then $\left\{x^{n}\right\}$ converges in norm to some point in $C$. In particular, this happens whenever there exist $\epsilon>0$ and a subsequence $\left\{n_{k}\right\}$ s.t. $\epsilon \leq \alpha^{\left(n_{k}\right)} \leq 2-\epsilon$ and $\hat{I}^{\left(n_{k}\right)}=\{1: N\}$ for all $k$.

Theorem 32 Suppose the algorithm is linearly focusing, $\hat{I}^{(n)}=\{1: N\}$ for all $n, 0<$ $\underline{\alpha}^{(\infty)}, \bar{\alpha}^{(\infty)}<2$ and $\left(C_{1}, \ldots, C_{N}\right)$ is boundedly linearly regular. Then $\left\{x^{n}\right\}$ converges linearly to some point in $C$; the rate of convergence is independent of the starting point whenever $\left(C_{1}, \ldots, C_{N}\right)$ is linearly regular.

To illustrate, we only quote one fact from (Bauschke and Borwein, 1995).

Fact 33 If $C_{\hat{\imath} \cap \operatorname{int}} \bigcap_{i \neq \hat{\imath}} C_{i} \neq \emptyset$ for some $\hat{\imath}$ then $\left(C_{1}, \ldots, C_{N}\right)$ is boundedly linearly regular.

\section{REFERENCES}

Aharoni, R. and Censor, Y. (1989) Block-iterative projection methods for parallel computation of solutions to convex feasibility problems. Linear Algebra and Applications, $120,165-75$.

Bauschke, H.H. and Borwein, J.M. (1995) On projection algorithms for solving convex feasibility problems. SIAM Review, (to appear).

Flåm, S.D. and Zowe, J. (1990) Relaxed outer projections, weighted averages and convex feasibility. BIT, 30, 289-300.

Kiwiel, K.C. (1995a) Block-iterative surrogate projection methods for convex feasibility problems. Linear Algebra and Applications, 215, 225-59.

Kiwiel, K.C. (1995b) The efficiency of subgradient projection methods for convex optimization, part II: Implementations and extensions. SIAM Journal on Control and Optimization, (to appear).

Kiwiel, K.C. and Lopuch, B. (1994) Surrogate projection methods for finding fixed points of firmly nonexpansive mappings. Systems Research Institute, Warsaw. 